\title{
MODELING OF FRACTURE PROPAGATION IN CONCRETE STRUCTURES USING A CONSTITUTIVE RELATION WITH EMBEDDED DISCONTINUITY*
}

\author{
STANISŁAW PIETRUSZCZAK, EHSAN HAGHIGHAT
}

McMaster University, Hamilton, Canada, e-mail: pietrusz@mcmaster.ca, haghige@mcmaster.ca

\begin{abstract}
In this paper, the problem of modeling of mixed mode cracking in concrete structures is addressed within the context of a constitutive law with embedded discontinuity (CLED). This approach, which was originally developed for describing the propagation of localized deformation in a "smeared" sense, is enhanced here to model a discrete nature of crack propagation. The latter is achieved by coupling the CLED approach with the level-set method, which is commonly used within the framework of Extended Finite Element (XFEM). Numerical simulations of experimental tests conducted at Delft University, which involve four-point bending of a notched concrete beam under the action of two independent actuators, are presented. The results based on enhanced CLED approach are directly compared with XFEM simulations. The predictions from both these methodologies are quite consistent with the experimental data, thereby giving advantage to CLED scheme in view of its simplicity in the numerical implementation.
\end{abstract}

Key words: XFEM, embedded discontinuity model, level set method, cohesive crack propagation

\section{INTRODUCTION}

In this study, the constitutive law with embedded discontinuity (CLED), which was first proposed in [1] and later modified in [2], is implemented to model the damage evolution in brittle materials. The original methodology is enhanced here by employing the level set method in order to capture the discrete nature of the macrocrack propagation.

Over the last few decades, intensive research has been conducted on modeling of propagation of brittle damage within the framework of finite element analysis. The early methodology was based on separating the element edges during crack formation process [3], [4] and was later combined with adaptive re-meshing approaches [5], [6]. An alternative approach that was widely used in the 1980's was the concept of smeared cracking that incorporated some plasticity based strain-softening relations [7], [8]. Such an approach was restrictive, in the sense that it would not allow for tracing discrete cracks and the solution was, in general, sensitive to discretization. In order to remedy this problem, a simple homogenization technique was introduced in [1], which incorporated a characteristic dimension associated with the geometry of the localized zone. This was later followed by the use of elements with embedded localization zones [9]. Afterward, element free Galerkin methods have been developed for modeling of crack propagation problems as they display higher accuracy compared to standard FEM interpolations [10].

Introduction of the partition of unity concepts [11], [12] has opened a new door in numerical modeling of discrete propagation of brittle damage and resulted in the development of the Extended Finite Element Method, XFEM, [13], [14]. The latter allows for incorporation of discontinuous displacement fields as well as the tip enrichments into the approximation space. More recently, the approach has been coupled with the level-set method for a more efficient tracing of the path of crack propagation [15]. In the last decade, XFEM has been used for modeling of discontinuities, within FEM approach, in a wide range of applications such as dynamic problems [16], hydromechanical problems [17], [18] as well as thermoelasticity problems [19].

This paper is focused on the modeling of mixed mode cracking in a class of brittle materials, like con-

\footnotetext{
* This paper was presented at the 8th International Conference on Analytical Models and New Concepts in Concrete and Masonry Structures AMCM 2014, Wrocław.
} 
crete. Both standard FEM, incorporating a constitutive law with embedded discontinuity (CLED), as well as XFEM are employed. The former methodology, i.e., CLED, is enhanced by coupling this approach with the level-set method for tracing the propagating macrocrack. A numerical example is provided, which deals with simulations of experimental tests conducted at Delft University [20]. These involve four-point bending of a notched concrete beam under the action of two independent actuators. The results based on enhanced CLED approach are directly compared with XFEM simulations. The predictions from both these methodologies are consistent with the experimental data, thereby giving advantage to CLED scheme in view of its simplicity in the numerical implementation. In particular, since CLED approach is a plasticity-based formulation, it can be easily incorporated in virtually any commercially available standard FEM package as it does not require the incorporation of any additional degrees of freedom.

The paper is structured as follows. In Section 2, the mathematical formulation is briefly reviewed for both XFEM and CLED methodologies. In Section 3, the strategy for monitoring the crack propagation is explained and a simple algorithm is proposed that can be used for any linear/nonlinear representation of the material behaviour. The details of numerical simulations are provided in Section 4.

\section{DISCONTINUOUS MOTION: XFEM VS. CONSTITUTIVE LAW WITH EMBEDDED DISCONTINUITY (CLED)}

A discontinuous motion, within a domain $\Omega$, can be described as

$$
v_{i}\left(x_{\alpha}, t\right)=\hat{v}_{i}\left(x_{\alpha}, t\right)+\mathcal{H}_{\Gamma_{d}} \widetilde{v}_{i}\left(x_{\alpha}, t\right)
$$

where the velocities $\hat{v}_{i}\left(x_{\alpha}, t\right)$ and $\tilde{v}_{i}\left(x_{\alpha}, t\right)$ are continuous functions, while $\mathcal{H}_{\Gamma_{d}}=\mathcal{H}\left(\phi\left(x_{\alpha}\right)\right)$ is the Heaviside function which defines the discontinuous motion along interface $\Gamma_{d}$ whose geometry is described by level-set function $\phi\left(x_{\alpha}\right)$. The gradient of equation (1) may be expressed as

$$
\begin{gathered}
v_{i, j}\left(x_{\alpha}, t\right)=\hat{v}_{i, j}\left(x_{\alpha}, t\right)+\mathcal{H}_{\Gamma_{d}} \widetilde{v}_{i, j}\left(x_{\alpha}, t\right) \\
+\delta_{\Gamma_{d}} \hbar \widetilde{v}_{i}\left(x_{\alpha}, t\right) \widetilde{n}_{j}\left(x_{\alpha}\right) .
\end{gathered}
$$

In this equation, $\delta_{\Gamma_{d}}=\delta(\phi(x))$ is the Dirac delta function that is singular at $\phi(x)=0$ and equal to zero elsewhere. Moreover, $\hbar$ represents the jump in the discontinuous function, i.e., $\hbar=\llbracket \mathcal{H}(\phi(x)) \rrbracket$, and $\widetilde{n}_{i}$ is the normal to the surface $\Gamma_{d}$. The velocity discontinuity along the interface $\Gamma_{d}, \dot{g}_{i}=\llbracket v_{i}\left(x_{\alpha}, t\right) \rrbracket$, can be defined viz. equation (1) as

$$
\dot{g}_{i}=\llbracket \hat{v}_{i}\left(x_{\alpha}, t\right)+\mathcal{H}_{\Gamma_{d}} \widetilde{v}_{i}\left(x_{\alpha}, t\right) \rrbracket=\hbar \widetilde{v}_{i}\left(x_{\alpha}, t\right) .
$$

Note that the jumps of continuous functions $\hat{v}_{i}$ and $\tilde{v}_{i}$ are zero. Using the definition (3), equation (2) can be re-written as

$$
\begin{gathered}
v_{i, j}\left(x_{\alpha}, t\right)=\hat{v}_{i, j}\left(x_{\alpha}, t\right)+\mathcal{H}_{\Gamma_{d}} \widetilde{v}_{i, j}\left(x_{\alpha}, t\right) \\
+\delta_{\Gamma_{d}} \dot{g}_{i}\left(x_{\alpha}, t\right) \widetilde{n}_{j}\left(x_{\alpha}\right) .
\end{gathered}
$$

Here, the first two terms appearing at the right hand side of equation (4) are associated with a motion in the neighborhood of the discontinuity, while the last term is valid only along the discontinuity due to the presence of the Dirac delta function.

\subsection{XFEM APPROACH}

Within the XFEM framework, the discontinuous motion can be directly incorporated into the finite element approximation space. Based on equation (1), XFEM approximation can be expressed as

$$
\begin{gathered}
v_{i}^{h}\left(x_{\alpha}, t\right)=\hat{v}_{i}^{h}\left(x_{\alpha}, t\right)+\mathcal{H}_{\Gamma_{d}} \widetilde{v}_{i}^{h}\left(x_{\alpha}, t\right) \\
=\sum_{I=1, \hat{n}} \hat{N}_{I}\left(x_{\alpha}\right) \dot{\hat{d}}_{I i}(t)+\sum_{I=1, \tilde{n}} \mathcal{H}\left(\phi\left(x_{\alpha}\right)\right) \tilde{N}_{I}\left(x_{\alpha}\right) \dot{\vec{d}}_{I i}(t)
\end{gathered}
$$

where $\hat{N}_{I}$ and $\widetilde{N}_{I}$ are standard finite element shape functions while $\dot{\hat{d}}_{I i}(t)$ and $\dot{\tilde{d}}_{I i}(t)$ are standard and enriched degrees of freedom associated with node $I$ and direction $i$, respectively.

\subsection{CONSTITUTIVE LAW WITH EMBEDDED DISCONTINUITY (CLED)}

Another way in which a discontinuous motion can be taken into account is through the constitutive relation, by introducing a discontinuous strain measure. This can be accomplished by decomposing the total strain rate into a continuous part, $\dot{\bar{\varepsilon}}_{i j}$, and a discontinuous one, $\dot{\widetilde{\varepsilon}}_{i j}$, viz.

$$
\dot{\varepsilon}_{i j}=\dot{\bar{\varepsilon}}_{i j}+\dot{\widetilde{\varepsilon}}_{i j}
$$


The continuous part represents the strain rates in the intact material, i.e., in the domain adjacent to the interface, while the discontinuous part is due to the motion along the discontinuity surface. The continuous part itself may be resolved into an elastic and a plastic component as $\dot{\bar{\varepsilon}}_{i j}=\dot{\bar{\varepsilon}}_{i j}^{e}+\dot{\bar{\varepsilon}}_{i j}^{p}$. Considering the analytical form defined by representation (4), there are two different terms describing the kinematic characteristics; one deals with continuous motion defined through $\bar{v}_{i, j}=\hat{v}_{i, j}+\mathcal{H} \widetilde{v}_{i, j}$ and the other is associated with the discontinuous motion along the interface, i.e., $\delta_{\Gamma_{d}}\left(\dot{g}_{i} n_{j}\right)$. It is clear that the continuous part corresponds to $\dot{\bar{\varepsilon}}_{i j}$, while the discontinuous part is related to development of velocity discontinuities in the interfacial material. It is evident from the nature of the Dirac Delta function that the latter part acts only along the interface. The discontinuous motion can now be distributed over the considered reference domain by averaging over the volume that includes the discontinuity $\Gamma_{d}$. Thus, the strain rates $\dot{\widetilde{\varepsilon}}_{i j}$, due to the presence of discontinuity, can be defined as

$$
\dot{\widetilde{\varepsilon}}_{i j}=\frac{1}{\Delta \mathrm{v}} \int_{\Delta \mathrm{v}} \delta_{\Gamma_{d}}\left(\dot{g}_{i} n_{j}\right)^{s} d \mathrm{v}=\frac{1}{\Delta \mathrm{v}} \int_{\Delta a}\left(\dot{g}_{i} n_{j}\right)^{s} d a
$$

where the superscript $s$ denotes the symmetric part of the dyadic operator, $\Delta \mathrm{v}$ is the given reference volume and $\Delta a$ is the area of the discontinuity. Considering the last integral to represent an average value of the dyadic product $\dot{g}_{i} n_{j}$ over the differential area $\Delta a$ and defining $\chi=\Delta a / \Delta \mathrm{v}$, one can approximate equation (7) as

$$
\dot{\widetilde{\varepsilon}}_{i j}=\frac{\Delta a}{\Delta \mathrm{v}}\left(\dot{g}_{i} n_{j}\right)^{s} .
$$

In conclusion, the strain decomposition in the presence of discontinuous motion can now be defined as

$$
\dot{\varepsilon}_{i j}=\dot{\bar{\varepsilon}}_{i j}+\dot{\widetilde{\varepsilon}}_{i j}=\dot{\bar{\varepsilon}}_{i j}+\chi\left(\dot{g}_{i} n_{j}\right)^{s} .
$$

Note that the representation (9) is identical to the strain decomposition introduced in [2].

It is evident that, in contrast to XFEM approach where additional degrees of freedom are required to define the discontinuous motion, viz. equation (5), the velocity discontinuity $\dot{g}_{i}$ is perceived here as an internal variable embedded in the constitutive law. Its value can be specified by following a standard plasticity procedure and imposing the traction continuity condition along the interface. Invoking the decomposition (9), one can write

$$
\dot{\sigma}_{i j}=D_{i j k l}\left(\dot{\varepsilon}_{k l}-\dot{\widetilde{\varepsilon}}_{k l}\right)=D_{i j k l} \dot{\varepsilon}_{k l}-D_{i j k l}\left(\chi \dot{g}_{k} n_{l}\right) .
$$

Now, imposing the continuity condition along the interface, i.e., $n_{i} \sigma_{i j}=t_{j}=K_{j i} \dot{g}_{i}$, and invoking equation (10), the following relation is obtained after some algebraic transformations

$$
\begin{gathered}
\dot{\widetilde{\varepsilon}}_{i j}=E_{i j p q} D_{p q k l} \dot{\varepsilon}_{k l} ; \\
E_{i j p q}=\chi n_{i}\left(K_{j p}+\chi n_{r} D_{r j p s} n_{s}\right)^{-1} n_{q} .
\end{gathered}
$$

Thus, the constitutive relation, which incorporates an embedded discontinuity, takes the form

$$
\dot{\sigma}_{i j}=\widetilde{D}_{i j k l} \dot{\varepsilon}_{k l} ; \quad \widetilde{D}_{i j k l}=D_{i j k l}-D_{i j p q} E_{p q r s} D_{r s k l} .
$$

Note that if the continuous part of the body is considered as elastic, which is the case in this study, there is $D_{i j k l}=D_{i j k l}^{e}$ and the material nonlinearity is embedded in the operator $K_{i j}$ which defines the interfacial properties.

\section{CRACK INITIATION AND PROPAGATION STRATEGY}

The main topic in this study is that of modeling of discrete crack propagation. As mentioned earlier, XFEM approach provides a convenient numerical tool for dealing with this class of problems. The key features here are the ability to trace the propagating crack through the level-set method and the mesh-independency of the solution. The latter, i.e., the meshindependency has always been an important issue in the context of the numerical modeling of damage propagation problems.

The approach based on constitutive law with embedded discontinuity (CLED), that was introduced in [1], [2], also provides a mesh-independent framework, however it was originally formulated as a smeared strategy for modeling of strain localization. Here, the original methodology is extended to modeling of discrete cohesive crack propagation problems. This is accomplished by coupling this approach with the level-set method that is employed in XFEM. This also helps in terms of defining the characteristic length parameter $\chi$ in the discontinuous strain measure.

In most works incorporating XFEM framework, the J-integral method is used to evaluate the stress intensity factors and the direction of crack propagation. However, the range of applicability of this approach is limited to elastic-brittle materials undergoing a tensile damage. A simple and effective way of 
defining the onset of fracture and the direction of the propagating crack is to invoke a specific form of the failure criterion. For failure in tension, for example, the maximum tensile stress criterion may be employed which stipulates that the direction of crack is perpendicular to that of major principal stress. In case of frictional materials, commonly described by Mohr -Coulomb criterion, the crack is also said to form at a prescribed orientation relative to the principal stress system. Note that for an elasto-plastic material, the direction of localization is often defined as a bifurcation problem.

It should be pointed out that the integral approaches, such as the J-integral method, have an implicit averaging nature; i.e., they provide average measures defined over a domain of interest. Using a similar concept of average property, a simple algorithm is implemented here for assessing the direction of propagation of the discontinuity surface.

In a typical scenario, the stress state at most integration points in the vicinity of the crack tip is close to the failure envelope. Therefore, the scheme used here for assessing the direction of propagation is based on checking the failure criterion at the integration points adjacent to the tip element. The average direction of propagation is then established based on the orientations associated with the neighboring integration points. This algorithm is illustrated schematically in Fig. 1. Note that by implementing this methodology, a stable crack pattern propagation is achieved for both XFEM and CLED, which is not the case without incorporation of the averaging scheme.

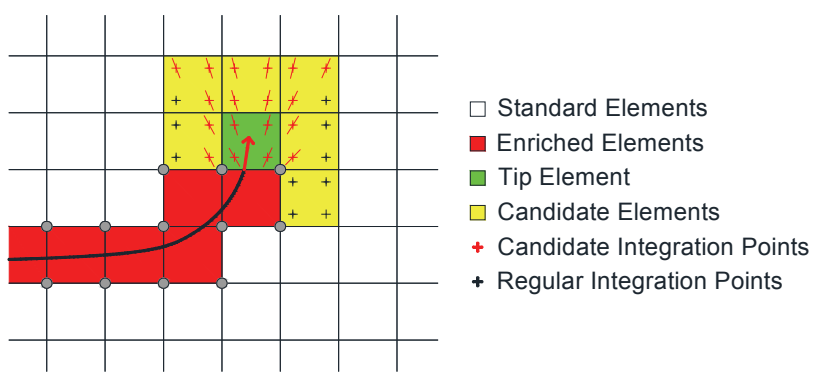

Fig. 1. Crack propagation methodology used for both XFEM and FEM/EDM

\section{NUMERICAL SIMULATIONS}

In this section, the experimental tests conducted by Galvez and his co-workers [20] at Delft University are simulated. The analysis incorporates a simple cohesive crack model with an exponential decay [21].
Within this framework, the tensile strength of the material is expressed as

$$
\mathcal{F}_{t}\left(g_{n}\right)=F_{t} \exp \left(\frac{-F_{t}}{G} g_{n}\right)
$$

where $F_{t}$ is the initial value at the onset of cracking, $G$ is a material constant which can be interpreted as the fracture energy release rate, and $\mathcal{F}_{t}\left(g_{n}\right)$ is the tensile strength at separation $g_{n}$. The yield criterion is written in the form

$$
f\left(t_{n}, g_{n}\right)=t_{n}-\mathcal{F}_{t}\left(g_{n}\right) .
$$

Thus, for an active loading process, setting $f\left(t_{n}, g_{n}\right)=0$ will result in $t_{n}=\mathcal{F}_{t}\left(g_{n}\right)$. Note that the representation (14) does not involve the tangential component of cohesive force, which is assumed here as negligible. Therefore, the constitutive relation for the interfacial material, referred to the local coordinate system along the crack, can be expressed as

$$
\dot{t}_{i}^{*}=K_{i j}^{*} \dot{g}_{j}^{*} ; \quad K_{i j}^{*}=\left\{\begin{array}{cc}
-\frac{F_{t}^{2}}{G_{f}} \exp \left(-\frac{F_{t}}{G_{f}} g_{n}\right) & i=j=1, \\
0 & \text { otherwise. }
\end{array}\right.
$$

The geometry of the problem analyzed here is shown in Fig. 2. The problem involves four-point bending of a notched concrete beam under the action of two independent actuators.

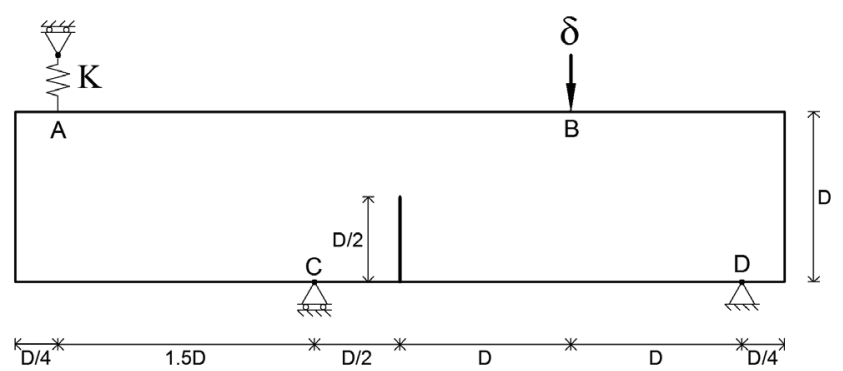

Fig. 2. Geometry and boundary conditions of the mixed mode cracking problem

In the original experimental studies, the specimens were tested at different values of $D$ and $K$. The simulations conducted here correspond to $D=300 \mathrm{~mm}$ and involve two limiting cases of the value of $K$, viz. $K=0$ (no constraint) and $K=\infty$ (point A fixed). Note that the problem was considered as plane-stress and was analyzed as displacement-controlled (viz. $\delta$ ). The material was considered to be brittle-elastic and the criterion for the onset of cracking was based on the critical value of the maximum tensile stress. The material properties were taken as follows 
a)
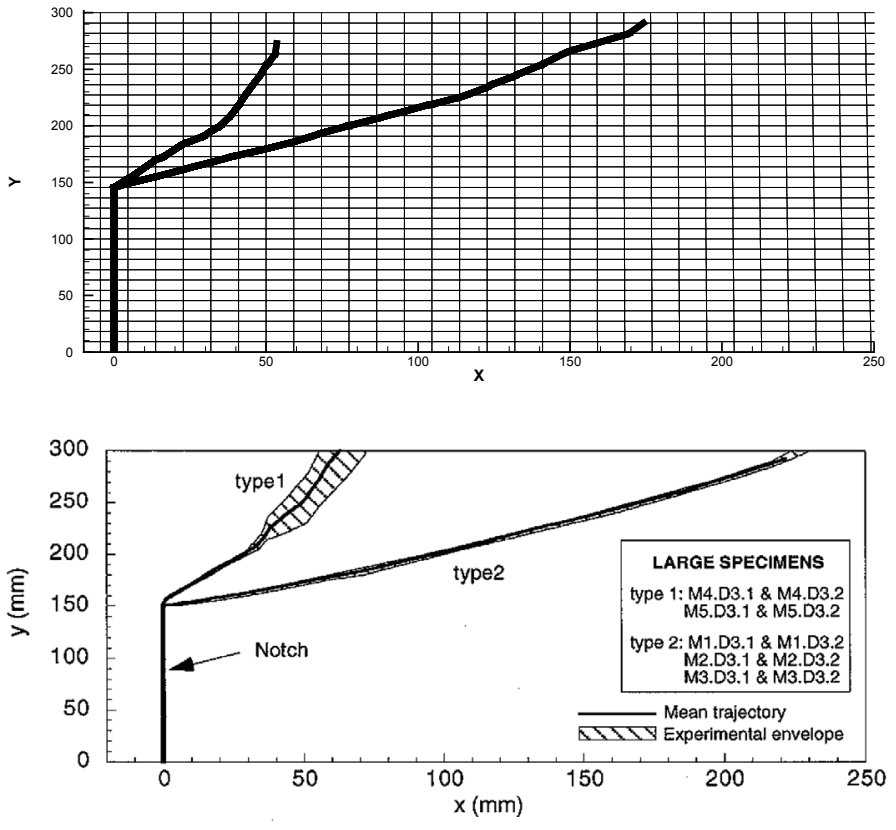

Fig. 3. Cracking pattern from (a) XFEM and FEM/CLED simulations and, (b) experiment
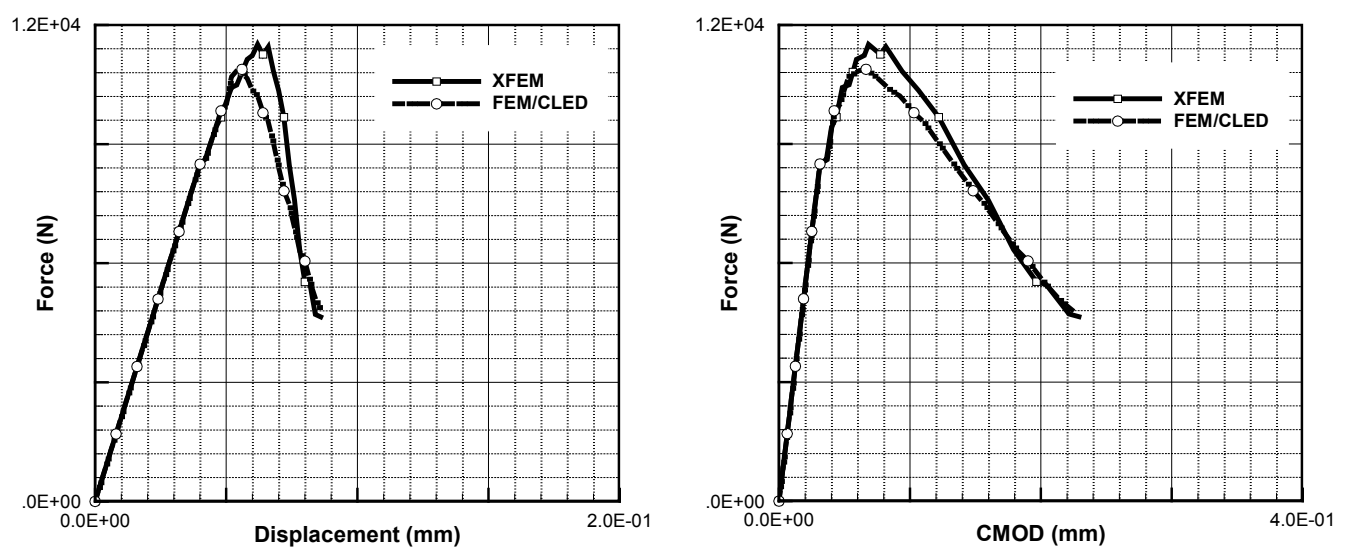

Fig. 4. Reaction force vs. applied displacement and crack mouth opening displacement curves for FEM/CLED and XFEM simulations

$$
\begin{gathered}
E=38 \mathrm{GPa}, \quad v=0.2, \\
F_{t}=3 \mathrm{MPa}, \quad G_{f}=70 \mathrm{~N} / \mathrm{m},
\end{gathered}
$$

while the thickness was assumed to be equal to $50 \mathrm{~mm}$.

The cracking pattern, for both cases considered in the analysis, is shown in Fig. 3, together with the relevant experimental data. Figure 4 presents the force-displacement diagrams, for both FEM/CLED and XFEM simulations, which correspond to $K=0$. These are supplemented by an image showing the scaled deformed shape (see Fig. 5). Figures 6 and 7 present the results of simulations corresponding to $K=\infty$. It is quite evident that the predictions using both methodologies are virtually identical and are fairly consistent with the experimental data reported in [20].

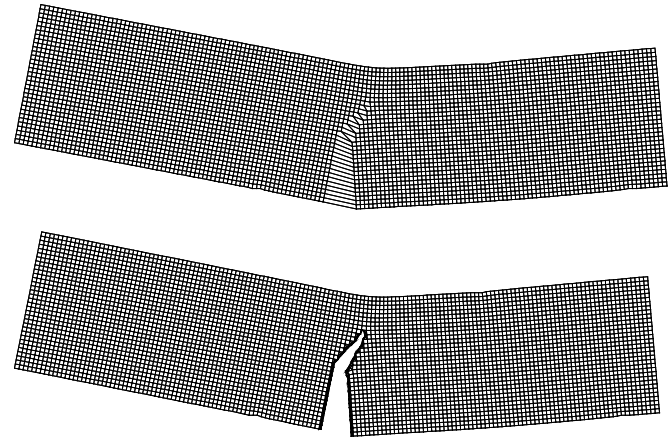

Fig. 5. Scaled deformed shape from FEM/CLED and XFEM simulations ( scale factor $=400$ ) 

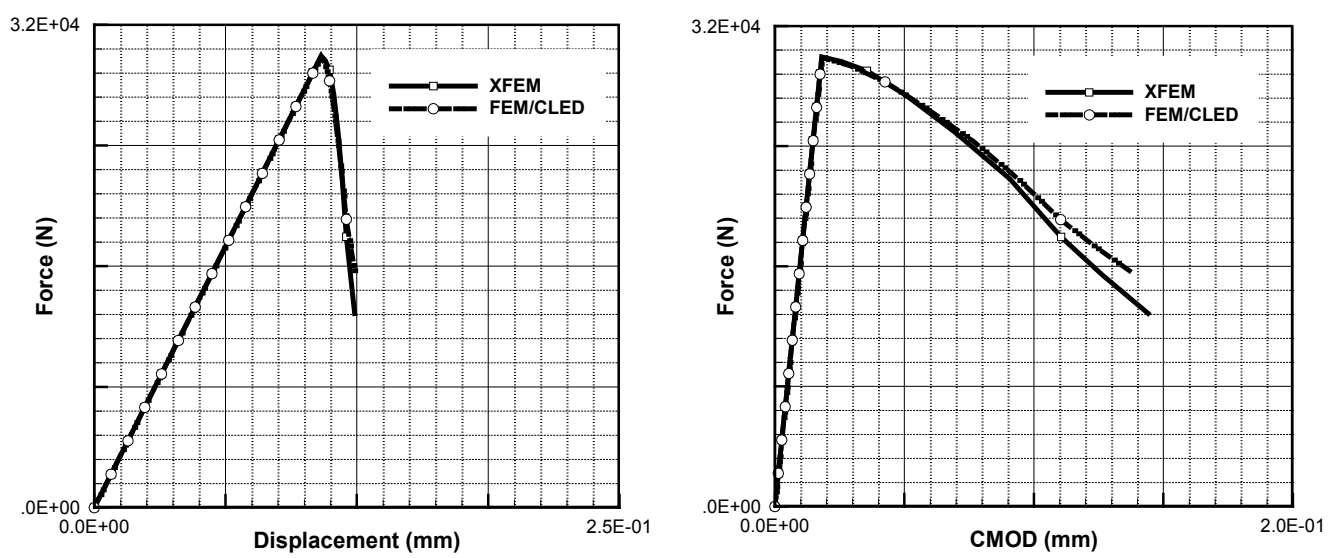

Fig. 6. Reaction force vs applied displacement and crack mouth opening displacement curves for FEM/CLED and XFEM simulations
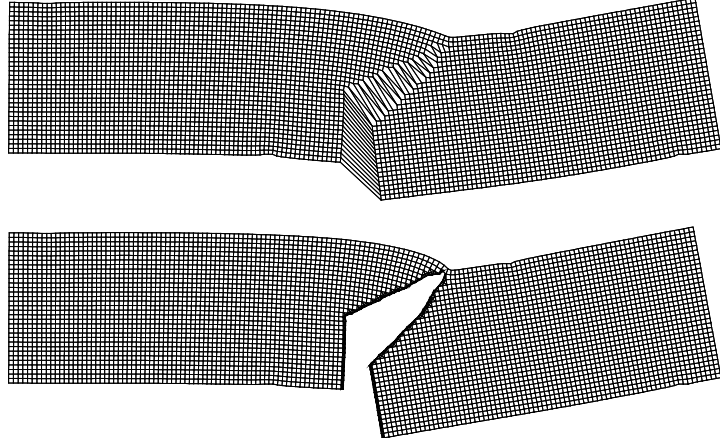

Fig. 7. Scaled deformed shape from FEM/CLED and XFEM simulations $($ scale factor $=400)$

\section{CONCLUDING REMARKS}

In this study, the problem of cohesive crack propagation in brittle materials has been investigated using two conceptually different methodologies, i.e., FEM/CLED and XFEM. The former approach, which is computationally more efficient, incorporates a constitutive law with embedded discontinuity. This approach has been coupled with the level-set method for the tracing of crack propagation process within the discretized domain. The Extended Finite Element Method (XFEM) has also been employed here, primarily for the purpose of examining the accuracy of the FEM/CLED approach.

The numerical analysis involved simulations of a four-point bending test conducted on a notched concrete beam [20]. The results of simulations using both methodologies, i.e., FEM/CLED and XFEM, were very similar in terms of both the evolution of damage pattern as well as the global load-displacement response. The results were also consistent with the experimental data.
The primary conclusion emerging from this study is that the classical constitutive model with embedded discontinuity, when enhanced with an appropriate scheme for tracing the crack propagation, provides a practical and accurate methodology for describing the localized deformation process.

\section{REFERENCES}

[1] Pietruszczak S., Mroz Z., Finite Element Analysis of Deformation of Strain-Softening Materials, Int. J. Numer Meth. Engng., 1981, Vol. 17, 327-334.

[2] PietruszcZaK S., On Homogeneous and Localized Deformation in Water-Infiltrated Soils, International Journal of Damage Mechanics, 1999, Vol. 8, 233-253.

[3] NGo D., SCORDElis A.C., Finite Element Analysis of Reinforced Concrete Beams, ACI Journal Proceedings, 1967, Vol. 64, $152-163$.

[4] NiLson A.H., Nonlinear Analysis of Reinforced Concrete by the Finite Element Method, ACI Journal Proceedings, 1968, Vol. 65, 757-66.

[5] Saouma V.E., Ingraffea A.R., Gergely P., White R.N., Interactive Finite Element Analysis of Reinforced Concrete: A Fracture Mechanics Approach, 1981.

[6] Shephard M.S., Yehia N., Burd G.S., Weidner T.J., Automatic Crack Propagation Tracking, Computers \& Structures, 1985, Vol. 20, No. 1, 211-223.

[7] NAYAK G.C., ZiENKIEWICZ O.C., Elasto-Plastic Stress Analysis: A Generalization for Various Constitutive Relations Including Strain Softening, Int. J. Numer Meth. Engng., 1972, Vol. 5, 113-135.

[8] BaŽAnt Z.P., Cedolin L., Blunt Crack Band Propagation in Finite Element Analysis, Journal of the Engineering Mechanics, 1979, Vol. 105, No. 2, 297-315.

[9] Belytshko T., Fish J., Engelmann B.E., A finite element with embedded localization zones, Computer Methods in Applied Mechanics and Engineering, 1988, Vol. 70, 59-89.

[10] BelYTShKo T., Lu Y.Y., Gu L., Element-free Galerkin methods, Int. J. Numer Meth. Engng., 1994, Vol. 37, 229-256.

[11] LiU W.K., Jun S., Zhang Y.F., Reproducing kernel particle methods, Int. J. Numer Meth. Fluids, 1995, Vol. 20, 1081-1106. 
[12] Melenk J.M., Babuška I., The Partition of Unity Finite Element Method: Basic Theory and Applications, Computer Methods in Applied Mechanics and Engineering, 1996, Vol. 139, 289-314.

[13] BelytschKo T., Black T., Elastic Crack Growth in Finite Elements with Minimal Remeshing, Int. J. Numer Meth. Engng., 1999, Vol. 45, 601-620.

[14] Moës N., Dolbow J., Belytschкo T., A Finite Element Method for Crack Growth Without Remeshing, Int. J. Numer Meth. Engng., 1999, Vol. 46, 131-150.

[15] Stolarska M., Chopp D.L., MoËs N., BelytschKo T., Modelling Crack Growth by Level Sets in the Extended Finite Element Method, Int. J. Numer Meth. Engng., 2001, Vol. 51, 943-960.

[16] Belytschko T., Chen H., Xu J., ZI G., Dynamic Crack Propagation Based on Loss of Hyperbolicity and a New Discontinuous Enrichment, Int. J. Numer Meth. Engng., 2003, Vol. 58, 1873-1905.
[17] Réthoré J., De Borst R., Abellan M.-A., A Two-Scale Approach for Fluid Flow in Fractured Porous Media, Int. J. Numer Meth. Engng., 2006, Vol. 71, 780-800.

[18] Khoei A.R., Haghighat E., Extended Finite Element Modeling of Deformable Porous Media with Arbitrary Interfaces, Applied Mathematical Modelling, 2011, Vol. 35, No. 11, 5426-5441.

[19] Areias P.M.A., Belytschko T., Two-Scale Method for Shear Bands: Thermal Effects and Variable Bandwidth, Int. J. Numer Meth. Engng., 2007, Vol. 72, 658-696.

[20] Gálvez J.C., Elices M., Guinea G.V., Planas J., Mixed Mode Fracture of Concrete Under Proportional and Nonproportional Loading, Int. J. Fract., 1998, Vol. 94, 267 284.

[21] Wells G.N., SLuYs L.J., A New Method for Modelling Cohesive Cracks Using Finite Elements, Int. J. Numer. Meth. Engng., 2001, Vol. 50, 2667-2682. 